\title{
A change in the outburst recurrence time of the Rapid Burster
}

\author{
N. Masetti ${ }^{\star}$ \\ Istituto Tecnologie e Studio della Radiazione Extraterrestre, CNR, via Gobetti 101, 40129 Bologna, Italy \\ Received 29 October 2001 / Accepted 20 November 2001

\begin{abstract}
An apparent sudden change in the outburst recurrence time displayed by the Rapid Burster occurred around the end of 1999 . The time between consecutive outbursts shrinked from $\approx 200$ to $\approx 100$ days somewhere between November 1999 and March 2000. In parallel, the average peak intensity also decreased of a factor $\sim 2$ in all outbursts occurred after November 1999 with respect to those occurred before this date. I discuss these results by comparing them with similar cases of changes in the recurrence time of other transient X-ray binaries and with the behaviour of Dwarf Novae. A viable explanation is connected with changes in the value of the quiescence mass transfer rate from the secondary, although the detailed origin of these changes remains at best speculative. Further, this result has important implications for programming time-constrained observations of the Rapid Burster both in outburst and in quiescence.
\end{abstract}

Key words. X-rays: binaries - stars: neutron - stars: individual: the Rapid Burster

\section{Introduction}

The Rapid Burster (MXB 1730-335; hereafter RB) is a well known and extensively studied low mass X-ray binary (LMXRB; see the review by Lewin et al. 1995) located in the globular cluster Liller 1, at a distance of $\sim 8 \mathrm{kpc}$ from earth (Ortolani et al. 1996). The RB is unique among LMXRBs in that it displays two different kinds of X-ray bursts: type I bursts, which are typical of many LMXRBs harbouring a low-magnetic field neutron star (NS), and type II bursts. The former events are interpreted as due to nuclear burning of accreted material on the surface of the NS. Type II bursts, instead, result from spasmodic accretion onto the NS surface (see e.g. Lewin et al. 1995). Types I and II burst emission patterns as observed with RXTE during several RB outbursts were thoroughly studied by Guerriero et al. (1999). Also, X-ray spectral analysis was carried out by Guerriero et al. (1999) with $R X T E$ during maximum and by Masetti et al. (2000) with BeppoSAX during the decay phase. Recently, a transient radio counterpart of the RB was observed by Moore et al. (2000) while the system was undergoing X-ray active phases, while the optical and infrared counterparts are still unknown or uncertain despite the high-accuracy position of the RB determined with Chandra (Homer et al. 2001).

Another characteristic feature of the RB is that this source is a recurrent transient with outbursts lasting a few weeks, followed by quiescent, or "off"-state, intervals which generally are $\sim 6-8$ months long (Lewin et al. 1993; Guerriero et al. 1999); thus far, no quantitative explanation for this behaviour has still been developed, although

\footnotetext{
* e-mail: masetti@tesre.bo.cnr.it
}

it may be possibly due to some accretion energy storage mechanism in the disk around the accreting NS as described by the disk-instability model (e.g. Lasota 2001). However, in this letter I will show, by means of the data collected up to now by the All-Sky Monitor (ASM) onboard RXTE, that recently this recurrence time has apparently undergone a substantial change. Section 2 will deal with the analysis of the ASM data, and in Sect. 3 a discussion of the results is given.

\section{ASM light curve data analysis}

The RXTE satellite (Bradt et al. 1993) carries an $\mathrm{ASM}^{1}$ (Levine et al. 1996) which regularly scans the $\mathrm{X}$-ray sky in the 2-12 keV range. During each scan, data from $90 \mathrm{~s}$ long dwells are obtained for each known X-ray source covered by the ASM. From these individual dwells (typically $5^{-}$ 10) a one-day averaged light curve of the monitored $\mathrm{X}$-ray sources with a daily sensitivity of $5 \mathrm{mCrab}$ is automatically computed and is available also. Figure 1 reports the complete up-to-date (November 2001) 2-12 keV ASM oneday averaged light curve of the RB starting on January 1996.

The light curve is divided into two panels for the sake of clarity in order to show an apparent change of recurrence time in the RB outbursts after MJD 51500 (November 17, 1999). It can also be noted that the outburst peak intensities are significantly lower after that date, and that the first two outbursts after that date, namely the one around MJD 51625 (March 21, 2000)

\footnotetext{
1 ASM light curves are available at: http://xte.mit.edu/ASM_lc.html
} 


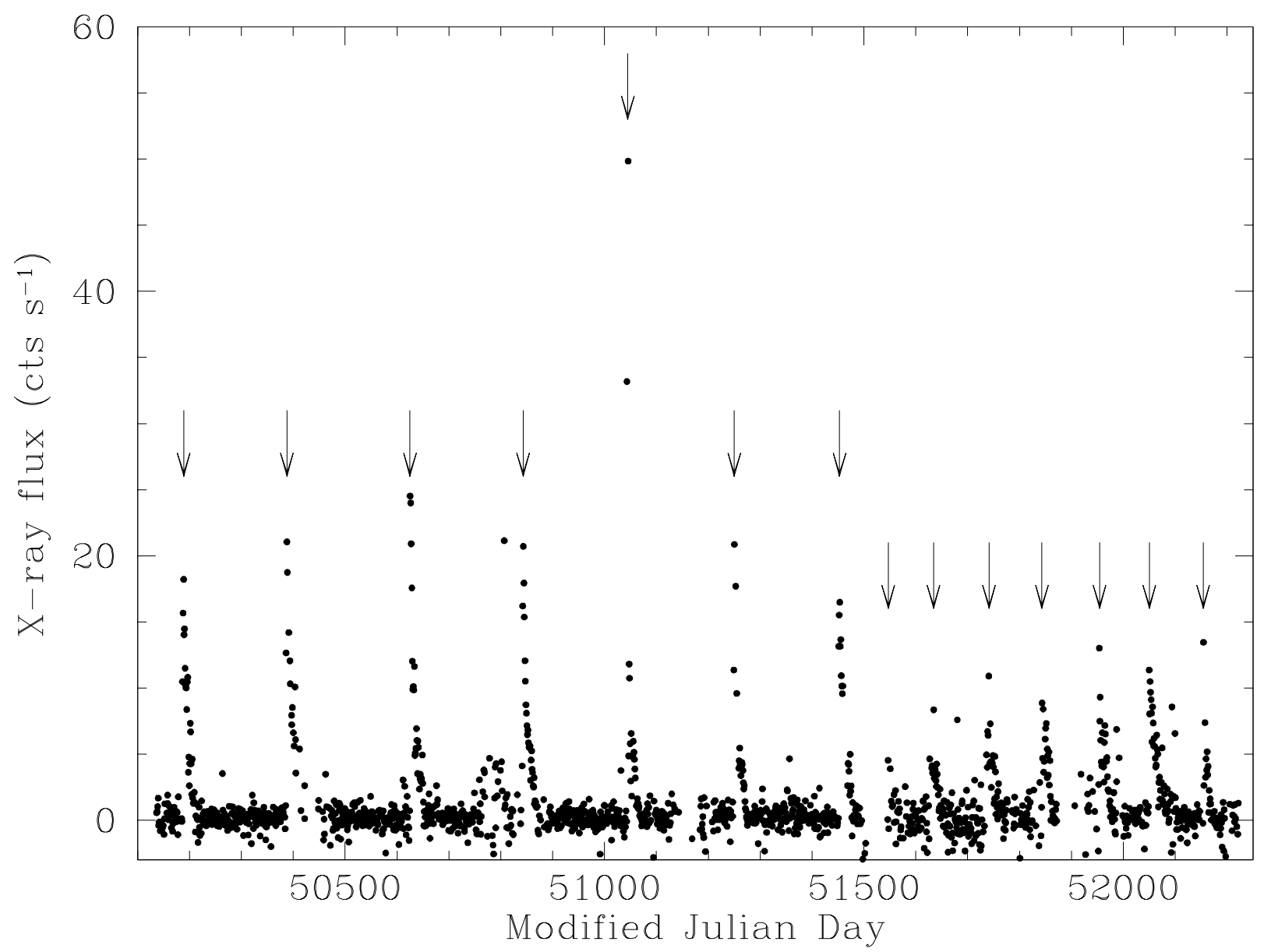

Fig. 1. 2-12 keV one-day averaged RXTE ASM light curve of the RB. 1 ASM count s ${ }^{-1}$ roughly corresponds to $13 \mathrm{mCrab}$ assuming a Crab-like spectrum. The reduced time interval between consecutive outbursts and the different average outburst peak intensities are apparent by comparing the data before and after MJD 51500 (i.e. 1999 November 17). The arrows indicate the times of outburst peaks. The errors associated with the data were not plotted in order to have a clearer view of the overall light curve shape.

and the previous, albeit less well determined, one around MJD 51550 (January 6, 2000), have the most scattered shape and the lowest peak intensity among the whole ASM set shown in Fig. 1.

To better study and to quantify this phenomenon, I performed a timing analysis of the entire RXTE ASM daily averaged data set using a Discrete Fourier Transform (DFT) algorithm (for a thorough description see Press et al. 1992). The Power Spectral Density (PSD) obtained with the DFT algorithm and corresponding to this data set is shown in Fig. 2. As one can see, the two most prominent peaks (marked by the arrows) fall at $0.48 \times 10^{-2}$ and $0.97 \times 10^{-2}$ cycles $\mathrm{d}^{-1}$, which correspond to $\sim 210$ and $\sim 100$ days, respectively.

In order to get a better insight on this result, and most of all to prove that the shorter recurrence periodicity of Fig. 2 is not simply an alias of the main one produced by the Fourier analysis of the ASM data set, I also ran the DFT algorithm on the data collected after MJD 51500. The corresponding PSD is reported in Fig. 3. From this figure it appears evident that only the peak corresponding to the higher frequency, here at $0.95 \times 10^{-2}$ cycles $\mathrm{d}^{-1}$
( $\approx 105$ days), has survived. Instead, at the position of the peak associated with the lower frequency (again marked with an arrow) there is no significant signal from the considered subset of data points.

Thus, the $\sim 6-8$ months long outburst recurrence time seems to have suddenly halved about two years ago and is still following this periodicity since then. The same can be said concerning the outburst average peak intensities, which changed from $\sim 25$ counts $\mathrm{s}^{-1}$ before November 1999 to $\sim 12$ counts $\mathrm{s}^{-1}$ after that date. Identical results are obtained if the DFT algorithm is applied to the dwell-bydwell ASM light curve of the RB.

Furthermore, to check the reliability of this result for the sake of completeness, I also inspected the periodicities displayed by the ASM data by running a non-DFT period searching algorithm based on the Phase Dispersion Minimization (PDM) method (Stellingwerf 1978). The results obtained with the PDM algorithm from the analysis of both the daily averaged and the dwell-by-dwell ASM data points do not show any significant difference with respect to those coming from the application of the DFT method. 


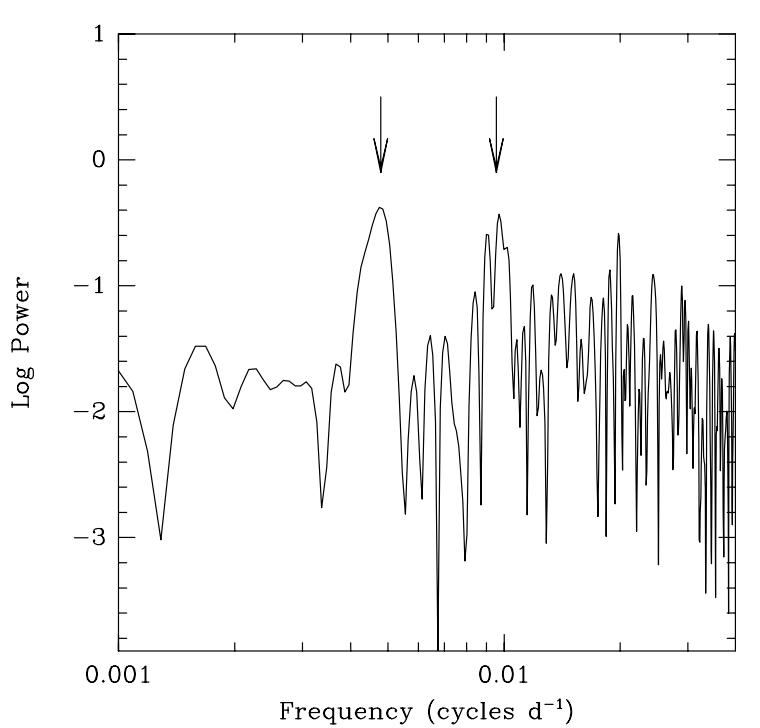

Fig. 2. PSD of the entire one-day averaged $R X T E$ ASM light curve of the RB shown in Fig. 1. The two most prominent periodicities at $\sim 210$ and $\sim 100$ days are indicated with the arrows.

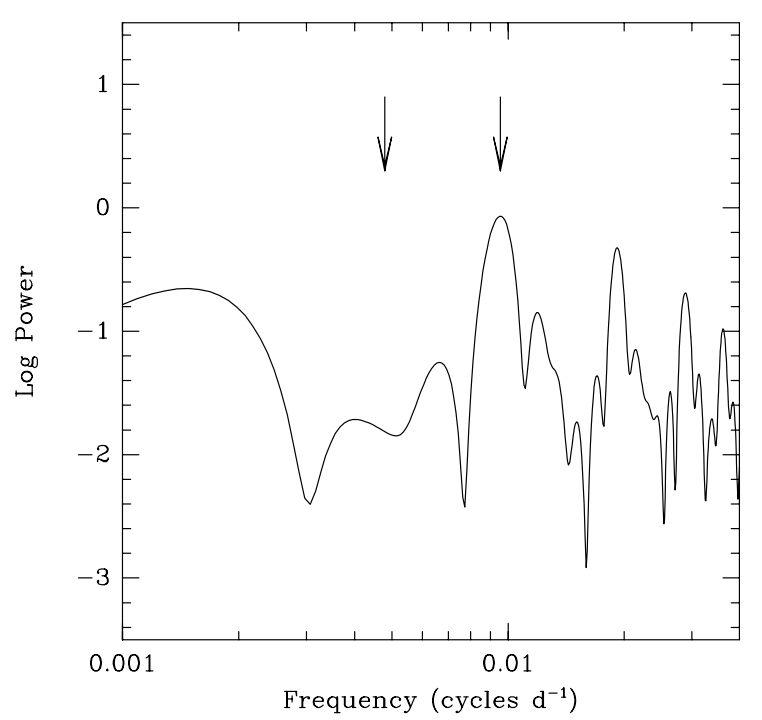

Fig. 3. The same as Fig. 2, but computed on the one-day averaged RXTE ASM data after MJD 51500. Only the shorter periodicity of Fig. 2 is present here, while no significant signal is detected at the position of the longer one.

\section{Discussion}

I have shown, from the analysis of the long-term monitoring of the RB by means of the RXTE ASM, that a change in the recurrence time of this source suddenly occurred around the end of 1999. In parallel, a decrease in the outburst peak intensity is seen occurring at the same time. Possibly, the first outburst (around MJD 51550) after this sudden change was aborted or at least not fully developed. Since then, the RB outbursts occurred following this new recurrence time.

Exploring the causes of this behaviour is not an easy task. As a first attempt, one can consider the possibility that the recurrence time reflects the orbital period of the system and that its change is connected with a modification in the orbit of the system. This is however unacceptable for several reasons. First, given that the RB is a LMXRB clearly hosting a NS (as it displays X-ray bursts) which is accreting via Roche lobe overflow, an orbital period of $\sim 200$ (or even of $\sim 100$ ) days is far too long to be reconciled with this scenario. Second, a reduction of $50 \%$ in the orbital period over a time comparable with the period itself was never seen in LMXRBs and very likely in any other type of close binary, and in any case it would indicate a severe orbital instability phase eventually leading to a very quick disruption of the system; no indication of all this is however apparent from the ASM data set shown in Fig. 1. Third, a long-period binary system would hardly survive the interactions occurring within the core of a globular cluster. Fourth, optical and near-infrared observations of the RB error box as determined by Chandra do not reveal the presence of any remarkable object with anomalous colors and/or resembling a giant (Homer et al. 2001). Therefore, the eventuality of a connection of the decrease in the recurrence time with a change in the supposed (long) orbital period of this system can reasonably be excluded.

An alternative, and in my opinion more viable, explanation may come by considering a sudden (altough possibly small) change in the average quiescence mass transfer rate $\dot{M}_{\mathrm{q}}$ from the secondary. Indeed, according to the diskinstability model, an increase in $\dot{M}_{\mathrm{q}}$ causes the recurrence time to shrink substantially (see e.g. Osaki 1996). An example of connection between these two parameters comes by considering the behaviour of SU UMa-type and ER UMa-type systems: both are short-period Dwarf Novae (DNe) in which a white dwarf is accreting from a late-type dwarf star via Roche lobe overflow, but objects belonging to the ER UMa-type group are characterized by shorter outburst cycles and smaller amplitude outbursts (Kato \& Kunjaya 1995). These differences are interpreted as due to the fact that $\dot{M}_{\mathrm{q}}$ from the secondaries in ER UMa-type systems is higher and closer to the critical value which marks the border between stable and unstable accretion disks (Osaki 1995, 1996).

Thus one may suppose that, given its frequent outbursts, the RB is close to the critical quiescence mass transfer rate $\dot{M}_{\mathrm{q}, \text { crit }}$ for (in)stability, and that an increase of $\dot{M}_{\mathrm{q}}$ may have brought the system even closer to persistence; this may have therefore started the production of outbursts with shorter recurrence times and lower peak intensities. It must however be noted that, in the case of the RB and more generally of LMXRBs, $\dot{M}_{\mathrm{q}}$ should not be confused with the total (quiescence plus outburst) mass transfer rate $\langle\dot{M}>$ averaged over an outburst cycle, which is an indicator the total energy emitted during the outburst, and in turn for the outburst peak intensity: looking at Fig. 1, one can argue that the value of this parameter has instead clearly reduced after MJD 51500. I also remark here that $\dot{M}$ in transient LMXRBs increases 
substantially during outburst because of strong X-ray irradiation of the secondary; this effect is instead practically absent in DNe due to the nature of the accreting object (van Paradijs 1996).

The trend observed in the light curve of Fig. 1 is also reminiscent of the behaviour followed by Type II bursts displayed by the RB during outbursts. Their time behaviour is like that of a relaxation oscillator: their fluence $E$ is roughly proportional to the time interval, $\Delta t$, to the following burst (Lewin et al. 1976) and their origin is known to be due to spasmodic accretion onto the NS (Lewin et al. 1993). Moreover, the long-term behaviour of the RB X-ray light curve is also similar to the well-known outburst pattern observed in the optical from DNe, in the sense that the longer is the time spent in quiescence after the last outburst, the larger will be the accretion energy stored in the disk and thus the more intense will be the subsequent outburst (for a complete phenomenological description of DNe, see Warner 1995). This is a further indication that an accretion phenomenon is responsible for the observed recurrence time change.

The reasons for this increase of $\dot{M}_{\mathrm{q}}$ are however not straightforward: in particular, it is not clear whether this is due to a increased $\mathrm{X}$-ray heating produced by the quiescent NS emission on the outer layers of the secondary star (as described by e.g. Hameury et al. 1986), or to a small change in the orbital parameters which shrinked the Roche lobe of the secondary star, or to other, more complex phenomena. It must be however stressed that a detailed analysis of the causes of this change in the RB outbursts recurrence time is beyond the scopes of this letter: here I just wanted to draw the reader's attention on the result as it is.

This variation in the outburst recurrence time, albeit peculiar, is actually not an unique behaviour among transient $\mathrm{X}$-ray binaries. Indeed, two recurrent Soft $\mathrm{X}$-ray Transients, namely 4U 1630-47 (suspected to harbour a black hole) and Aql X-1 (known to contain a NS) have shown changes in their outburst recurrence times in the past. Concerning 4U 1630-47, Kuulkers et al. (1997) found that the quiescent period between outbursts increased in a linear fashion from $\sim 600$ to $\sim 700$ days between the years 1984 and 1987. As regards Aql X-1, Kitamoto et al. (1993) noted that the outburst recurrence time increased substantially over the years, going from 125 days as observed between 1969 and 1979 to 309 days in the period 1987-1992. In both cases, and in particular for Aql X-1, changes in the value of $\dot{M}_{\mathrm{q}}$ from the secondary (possibly induced by chromospheric activity) or instabilities in the accretion disk were invoked to explain the observed behaviour, although none of these modelizations seems to satisfactorily describe these apparent changes in the "internal clock" of these systems (e.g. Kitamoto et al. 1993).

As a final remark, it should be noteworthy to say that the result illustrated here can also be useful for programming future multiwavelength observations of the next outbursts coming from the RB. Indeed, if this new recurrence time shown here for the RB outbursts will be maintained in the future, I expect that the next RB outburst will start around the last decade of December 2001. Moreover, a deep $\mathrm{X}$-ray observation of the RB in quiescence performed with high-sensitivity instruments onboard last-generation satellites such as Chandra and XMM-Newton is highly desireable in order to provide a high precision measurement of the quiescent flux from this system and a comparison with the only quiescent $\mathrm{RB}$ detection obtained with $A S C A$ - so far (Asai et al. 1996). Such a comparison will help to give a better insight on the proposed inrerpretation (the increase of $\dot{M}_{\mathrm{q}}$ ) as the cause of the observed change in the RB outburst recurrence time.

Acknowledgements. I would like to thank Filippo Frontera, Sergio Campana and Luigi Stella who triggered this analysis; I also thank Eliana Palazzi and again Luigi Stella for several important comments on the manuscript. I am grateful to the anonymous referee for several comments which helped me to improve this letter. ASM data were provided by the $R X T E$ ASM teams at MIT and at the RXTE SOF and GOF at NASA's GSFC.

\section{References}

Asai, K., Dotani, T., Kunieda, H., \& Kawai, N. 1996, PASJ, 48, L27

Bradt, H. V., Rothschild, R. E., \& Swank, J. H. 1993, A\&AS, 97, 355

Guerriero, R., Fox, D., Kommers, J., et al. 1999, MNRAS, 307, 179

Hameury, J. M., King, A. R., \& Lasota, J.-P. 1986, A\&A, 162,71

Homer, L., Deutsch, E. W., Anderson, S. F., \& Margon, B. 2001, AJ, 122, 2627

Kato, T., \& Kunjaya, C. 1995, PASJ, 47, 163

Kitamoto, S., Tsunemi, H., Miyamoto, S., \& Roussel-Dupre, D. 1993, ApJ, 403, 315

Kuulkers, E., Parmar, A. N., Kitamoto, S., Cominsky, L. R., \& Sood, R. K. 1997, MNRAS, 291, 81

Lasota, J.-P. 2001, New Astron. Rev., 45, 449

Levine, A. M., Bradt, H. V., Cui, W., et al. 1996, ApJ, 469, L33

Lewin, W. H. G., Doty, J., Clark, G. W., et al. 1976, ApJ, 207, L95

Lewin, W. H. G., van Paradijs, J., \& Taam, R. E. 1993, Space Sci. Rev., 62, 223

Lewin, W. H. G., van Paradijs, J., \& Taam, R. E. 1995, in X-ray Binaries, ed. W. H. G. Lewin, J. van Paradijs, \& E. P. J. van den Heuvel (Cambridge Univ. Press), 175

Masetti, N., Frontera, F., Stella, L., et al. 2000, A\&A, 363, 188

Moore, C. B., Rutledge, R. E., Fox, D. W., et al. 2000, ApJ, 532,118

Ortolani, S., Bica, E., \& Barbuy, B. 1996, A\&A, 306, 134

Osaki, Y. 1995, PASJ, 47, L11

Osaki, Y. 1996, PASP, 118, 39

Press, W. H., Teukolsky, S. A., Wetterling, W. T., \& Flannery, B. P. 1992, Numerical Recipes (Cambridge Univ. Press, Cambridge)

Stellingwerf, R. F. 1978, ApJ, 224, 953

van Paradijs, J. 1996, ApJ, 464, L139

Warner, B. 1995, Cataclysmic Variable Stars (Cambridge Univ. Press, Cambridge) 